\title{
SOLUCIÓN INTEGRADA DE CONTROL BASADO EN EMG PARA LA REHABILITACIÓN DE TERAPIAS BILATERALES EN EL EXOESQUELETO DE REHABILITACIÓN DE MANO ROBHAND
}

\author{
Ana Cisnal, David Sierra, Javier Pérez-Turiel, Juan Carlos Fraile \\ ITAP - Instituto de las Tecnologías Avanzadas de la Producción, Universidad de Valladolid. \\ \{ana.cisnal, david.sierra@uva.es ; \{jcfraile,jpturiel\}@eii.uva.es
}

\begin{abstract}
Resumen
Se ha demostrado que la rehabilitación bilateral asistida ayuda a los pacientes a mejorar su capacidad del miembro parético y promover su recuperación motora tras sufrir un accidente cerebrovascular (ACV), especialmente en las extremidades superiores. La rehabilitación bilateral asistida por robot, basada en el control por EMG, ha sido abordada anteriormente en otros estudios para mejorar la movilidad de la mano, pero faltan soluciones integradas de bajo coste para el control biocooperativo en tiempo real de las plataformas de rehabilitación robótica. Este artículo presenta una solución integrada de EMG en tiempo real de bajo coste, de diseño propio, implementada en la plataforma RobHand (Robot para la Rehabilitación de la Mano), un exoesqueleto que soporta terapias bilaterales basadas en EMG. Se ha desarrollado un control por umbrales basado en EMG para RobHand, que detecta el gesto de la mano sana y lo replica en el exoesqueleto colocado en la mano parética.
\end{abstract}

Palabras clave: Control, Electromiografía, Exoesqueletos, Neurorehabilitación, Robótica asistencial.

\section{INTRODUCCIÓN}

Los accidentes cerebrovasculares (ACV) son la tercera causa de discapacidad a nivel mundial, y afectan principalmente a los individuos en la cima de su vida productiva [8]. Aproximadamente el $75 \%$ de las personas sufren algún grado de paresia en los miembros superiores [14], que afecta especialmente a la mano [6].

Los pacientes con ictus mejoran el rendimiento motor de la mano mediante rehabilitación, que lleva al paciente a mejorar especialmente la amplitud de movimiento y la fuerza de la mano [16]. Sin embargo, la rehabilitación tradicional es muy costosa, ya que requiere mucho tiempo del especialista en rehabilitación. Un sistema robótico de rehabilitación que permitiese a los pacientes realizar el ejercicio repetitivo sin la asistencia continua del terapeuta, haría que la rehabilitación fuera más accesible y asequible. Por este motivo, los sistemas robóticos de rehabilitación de la mano han aparecido recientemente como un enfoque complementario al de las terapias tradicionales.

En los últimos años, el entrenamiento robótico bilateral ha ganado popularidad [9], [18], [19]. En las terapias bilaterales se replica el movimiento de referencia que produce la mano sana en el exoesqueleto acoplado a la mano dañada. Un control basado en señales electromiográficas (EMG) puede utilizarse para la realización de terapias bilaterales, determinando el movimiento de referencia a través del análisis de las señales EMG.

En este artículo se presenta una solución integrada de EMG en tiempo real implementada en RobHand, un exoesqueleto de rehabilitación de mano orientado a restaurar la capacidad de abrir y cerrar la mano de pacientes que han sufrido un accidente cerebrovascular. El control propuesto permite la realización de terapias bilaterales, en las que el exoesqueleto puesto en la mano parética se mueve de acuerdo a un control de umbrales que utiliza las señales EMG registradas de los músculos del antebrazo de la mano sana. Las terapias bilaterales se realizan utilizando entornos virtuales, o juegos serios, para aumentar la motivación del paciente.

El objetivo del proyecto es desarrollar una plataforma de rehabilitación de mano asequible y compacta, que pueda ser utilizada en el entorno doméstico. El coste de todo el sistema de rehabilitación se reduce utilizando actuadores de bajo coste y diseñando un sistema de adquisición de señales EMG a medida. En este trabajo se presenta una solución compacta y de bajo coste, consistente en un microcontrolador y un circuito para la adquisición de señales EMG, que proporciona una solución embebida para el exoesqueleto de mano.

La plataforma de rehabilitación robótica RobHand incluye un exoesqueleto de mano (sección 2), el sistema electrónico embebido (sección 3) en el que se 
ejecuta el control basado en EMG (sección 4) y un entorno software (sección 5).

\section{EXOESQUELETO DE MANO}

El exoesqueleto RobHand se basa en un mecanismo de cuatro barras en serie, de accionamiento directo y subactuado, que asiste a la flexión y extensión de los dedos de la mano [11]. Se utilizan cinco actuadores lineales L12-30-100-6-I (Actuonix Motion Devices Inc.), de $30 \mathrm{~mm}$ longitud de vástago y de bajo coste que proporcionan hasta $23 \mathrm{~N}$, para transmitir el movimiento a las falanges distal y proximal de cada dedo, cuyo movimiento está cinemáticamente acoplado (Fig. 1).

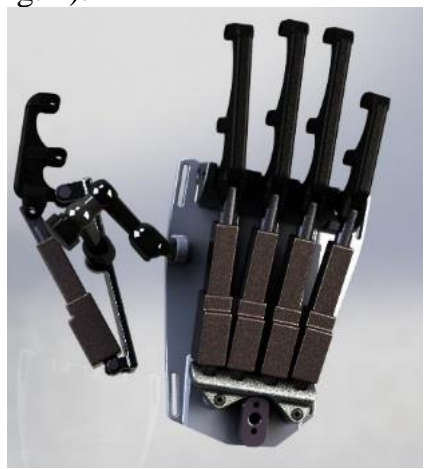

Figura 1: Diseño asistido por ordenador del exoesqueleto.

El uso de este mecanismo de cuatro barras en serie (four-bar linkage) reduce el tamaño y peso del exoesqueleto, pero sigue proporcionando los ángulos de flexión y extensión de una mano humana sana. Los enlaces transmiten el movimiento del motor a los anillos que vinculan la estructura mecánica a las falanges proximales y mediales de la mano. Los anillos son de material flexible (Filaflex 82a), lo que mejora la ergonomía del dispositivo y permite que se adapte a diferentes tamaños de dedos. Además, los anillos facilitan el procedimiento de colocación y retirada del exoesqueleto. La posición del pulgar es fácilmente ajustable a través de un mecanismo manual (dispositivo comercial Noga LC6200). El exoesqueleto está fabricado en aluminio, pesa unos 600 g. y se utiliza junto con un soporte de antebrazo para mitigar los efectos negativos generados por el propio peso del exoesqueleto.

\section{SISTEMA ELECTRÓNICO}

Se ha diseñado un circuito para adquirir y realizar el acondicionamiento analógico de las señales de la actividad eléctrica muscular resultante (señales electromiografías), registradas por electrodos de superficie. Además, este circuito transmite las señales EMG a un microcontrolador, configurado para realizar operaciones de procesamiento en tiempo real. El microcontrolador realiza el tratamiento digital de las señales para desarrollar el control basado en EMG y aplicar las señales de control adecuadas a los actuadores, para mover el exoesqueleto de acuerdo con la intención de movimiento del usuario.

Trabajos previos relacionados [1], [2], [3], [10], [12], [13], [15], [20] presentaban dos inconvenientes: alto coste del sistema debido a la utilización de sistemas de adquisición de señales EMG comerciales (solo [15] utiliza sistemas de bajo coste, considerados como aquellos que cuesta menos de $150 €$ ) y ejecución del procesamiento de las señales y del control en un ordenador, lo que puede incrementar el tiempo de latencia total del sistema. Teniendo en cuenta lo anteriormente expuesto, el sistema electrónico embebido desarrollado se ha diseñado para reducir el coste y el tiempo de latencia del sistema al máximo. Para ello, la solución presentada tiene como principales características que el sistema de adquisición de EMG es de diseño propio y se ha minimizado su coste en la medida de lo posible, con un precio inferior a $50 €$ para producción a pequeña escala, e incorpora un microcontrolador para el procesamiento a bordo y en tiempo real, que recibe los datos de EMG justo después de la conversión analógico-digital y que aplica la señal de control a los actuadores justo después de procesar los datos de EMG, de modo que el tiempo de latencia del sistema global se reduce.

En esta sección se presenta una solución embebida compacta de bajo coste para el control en tiempo real del exoesqueleto de mano accionado por EMG. Esta solución se compone principalmente de un microcontrolador TMS320F28069M (Texas Instruments, Texas, EEUU), un sistema de adquisición de EMG y una PCB para el control de los motores. Estos componentes (Fig. 2) y se detallan en los siguientes apartados.

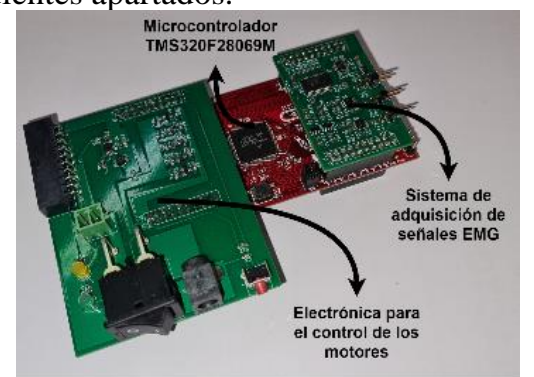

Figura 2. Componentes de la solución.

\subsection{MICROCONTROLADOR}

El microcontrolador (MCU) utilizado es el TMS320F28069M (Texas Instruments, Texas, EEUU) y tiene dos núcleos acoplados, el núcleo C28x y el CLA (Control Law Accelerator). El CLA ejecuta el código independientemente del núcleo 
C28x y se comunica con él mediante un bus de datos especializado (Bus CLA). Esta característica permitirá que la CPU principal se encargue de la comunicación y el control de los actuadores, mientras que el núcleo CLA se encargará únicamente del procesamiento de las señales de EMG. De esta manera, se consigue aumentar el ancho de banda del microcontrolador.

\subsection{SISTEMA DE ACQUISICIÓN DE SEÑALES EMG}

Se ha diseñado y desarrollado un circuito para la adquisición de señales electromiográficas de dos canales diferenciales de 24 bits de resolución y 112 DB de rango dinámico (DR). Cada canal consta de un amplificador de instrumentación seguido de un filtro RC de paso bajo. Para evitar la saturación del amplificador de instrumentación, cada canal está diseñado para compensar el offset de la entrada diferencial. El conversor analógico-digital está configurado para funcionar a una frecuencia de muestreo de 3,3 MHz y la relación de sobremuestreo está ajustada a 4096, con lo que cada canal muestrea las señales a una frecuencia de $200 \mathrm{~Hz}$. El circuito está diseñado para interactuar con el MCU TMS320F28069M, a través de una comunicación SPI y otras señales digitales necesarias.

La placa está diseñada en una PCB de 4 capas con un único plano de tierra (situado una capa interna) en la circuitería analógica y dos planos de tierra (superior e inferior) en la circuitería digital, de forma que los planos de tierra se dividen para separar los circuitos analógicos y digitales, mientras que el resto de capas son para las líneas de señal. La placa tiene un diseño de tipo "boosterpack" para evitar el uso de cables de conexión. Los componentes discretos se colocan en las capas superior e inferior para reducir aún más el tamaño de la placa resultante, que es de 50,8×33 mm. El circuito ocupa una superficie activa de $10 \mathrm{~cm}^{2}$ y consume $3 \mathrm{~mW}$, siendo alimentado a 3,3 V.

\subsection{CONTROLADORA DE LOS MOTORES}

El exoesqueleto cuenta con cinco actuadores lineales L12-30-100-6-I que se alimentan con DC 6V y tienen incorporado un controlador de posición interno que permite controlar la posición mediante el uso del modo PWM con el interfaz 0-5V, aplicando una señal de entrada PWM de $5 \mathrm{~V}$ y $1 \mathrm{KHz}$ cuyo ancho de pulso es proporcional al porcentaje de la extensión del vástago del actuador. Los actuadores están controlados por el MCU TMS320F28069M, que genera señales de salida PWM de 3,3V, por lo que es necesario diseñar y desarrollar un driver para los motores (un circuito electrónico que actúe como puente entre el microcontrolador y los actuadores), que eleve el nivel de tensión de la señal de salida
PWM de 3,3 a 5V, para cumplir las especificaciones del control en modo PWM.

El circuito impreso incluye cinco drivers para controlar los cinco actuadores de forma independiente. Cada controlador de motor es un circuito de subida de tensión, que tiene una etapa de amplificación compuesta principalmente por un transistor MOSFTET de canal N BSS138.

La placa controladora de los motores cuenta con un único conector para los cinco actuadores, por lo que facilita la conexión del exoesqueleto de mano a la caja electrónica. También cuenta con un botón de reset para el microcontrolador, un conector de alimentación de 6V DC en serie con un interruptor de encendido/apagado para permitir la conexión/desconexión de la alimentación y dos leds para la indicación visual del estado de alimentación del microcontrolador y de la fuente de alimentación. Por razones de seguridad, también tiene un pulsador de parada de emergencia, que puede ser utilizado para cortar la energía a los actuadores. La placa de control del motor desarrollada es una PCB de 2 capas, con componentes discretos sólo colocados en la capa superior.

\section{CONTROLADOR BASADO EN EMG}

El control planteado determina el movimiento del exoesqueleto basándose en las señales EMG registradas de los músculos responsables del movimiento de apertura y cierre de la mano.

\subsection{SELECCIÓN DE LOS MÚSCULOS Y COLOCACIÓN DE LOS ELECTRODOS}

La detección del movimiento de la mano, en concreto, de la apertura y el cierre de los dedos de la mano, es necesaria para controlar el exoesqueleto. Esta detección se consigue registrando las señales EMG de los músculos responsables de la flexión y extensión de los dedos de la mano. En concreto, se registran las señales EMG de los músculos Extensor Digitorum (ED) y Flexor Digitorum Superficialis (FDS), responsables de la apertura y el cierre de la mano, respectivamente. Se colocan dos pares de electrodos de superficie (Lessa -AB Medica Group, con un área de contacto de 30 x $30 \mathrm{~mm}$ ) en la superficie cutánea del vientre de los músculos objetivo con una distancia central de $3 \mathrm{~cm}$ (recomendada por SENIAM), mientras que el electrodo de referencia se fija en la superficie cutánea del olécranon.

\subsection{REGISTRO Y NORMALIZACIÓN DE LAS SEÑALES EMG}


Las señales EMG deben normalizarse para comparar la actividad EMG entre los músculos [7]. La normalización de la EMG es importante debido a las interferencias que afectan a las señales, como el ruido de la línea eléctrica, la transpiración de la piel, la impedancia de contacto del sensor, que provoca una gran variabilidad de la señal, y la interferencia cruzada de los músculos activos. Además, las pequeñas diferencias en la colocación de los electrodos entre sesiones comprometen la repetibilidad de las señales EMG [4]. El procesamiento de la EMG convierte la señal EMG bruta en una señal normalizada en el rango de [0, 100], donde un valor de 100 representa la contracción voluntaria máxima (MVC) del músculo. La normalización realiza sobre la señal EMG rectificada.

Las señales se registran mediante el sistema de adquisición de EMG presentado, con una frecuencia de muestreo de $200 \mathrm{~Hz}$. Antes de la conversión analógica-digital, las señales EMG sufren un acondicionamiento previo: las señales se amplifican con una ganancia de 50, y luego pasan por un filtro paso bajo de $150 \mathrm{~Hz}$. Tras su digitalización, se filtran con un filtro eliminador de banda (frecuencia central de $50 \mathrm{~Hz}$ y factor Q de 20) y un filtro FIR de paso alto (frecuencia de banda de parada de $0,01 \mathrm{~Hz}$, frecuencia de banda de paso de $10 \mathrm{~Hz}$, atenuación mínima de la banda de parada a $80 \mathrm{~dB}$ y ondulación máxima de la banda de paso de $0,1 \mathrm{~dB}$ ) para eliminar las interferencias electromagnéticas. Las señales filtradas se rectifican calculando la raíz cuadrada media (RMS) con una ventana de 10 puntos y luego se aplican a un filtro FIR de paso bajo (frecuencia de la banda pasante de $1 \mathrm{~Hz}$, frecuencia de la banda de parada de $2 \mathrm{~Hz}$, ondulación máxima de la banda pasante de $4 \mathrm{~dB}$ y atenuación mínima de la banda de parada de $10 \mathrm{~dB}$ ) para producir una representación más suave de la señal envolvente. La señal rectificada resultante (rEMG) tiene un periodo de 20 ms y se normaliza con respecto a los valores de MVC del paciente medidos en la calibración. La Fig. 6 (arriba y centro) muestra las señales EMG sin procesar y sus correspondientes señales normalizadas, de los músculos FDS y ED.

\subsection{CALIBRACIÓN}

Es necesario realizar una calibración antes de comenzar una sesión de rehabilitación para calcular los umbrales necesarios para el control basado por EMG y para determinar el MVC, que es necesario para normalizar la señal de EMG.

Se pide a los pacientes que realicen una calibración que consiste en relajar la mano y en realizar la máxima flexión y extensión de los dedos durante ocho segundos, mientras la acción a realizar y la actividad muscular se muestran al sujeto en la pantalla de un ordenador. Para eliminar los efectos transitorios entre los tres gestos de la mano (reposo, apertura, cierre), se desestiman dos segundos al principio y al final de cada gesto y sólo se consideran los cuatro segundos intermedios para determinar los umbrales (Fig. 3).

Durante la sesión de calibración se determinan dos valores de umbrales EMG ( $\mu$ y $\varepsilon$ ), que son los valores límite máximos correspondientes a la desactivación muscular más una constante de 0,1 . El umbral $\mu$ es el umbral del músculo FDS y $\varepsilon$ para el músculo ED. Los gráficos superior y central de la Fig. 6 muestran los umbrales mencionados.

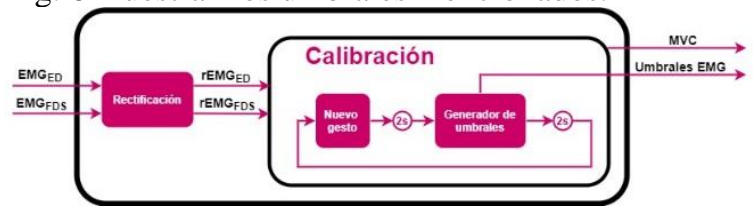

Figura 3: Diagrama de la fase de calibración

\subsection{CONTROL POR UMBRALES}

El control por umbrales basado en EMG permite realizar una terapia bilateral, reconociendo el gesto realizado por la mano sana y replicando ese gesto en el exoesqueleto colocado en la mano parética (Fig. 4). Para ello, se definen tres posibles gestos del exoesqueleto: "mano en reposo", "mano abierta" y "mano cerrada".

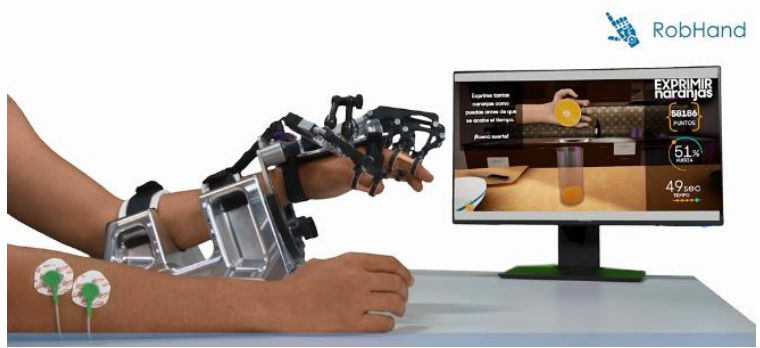

Figura 4: Realización de una terapia bilateral basada en EMG utilizando un entorno virtual

El funcionamiento del control por umbrales basado en EMG es el siguiente: el microcontrolador procesa y normaliza las señales de EMG registradas de los dos músculos del antebrazo de la mano sana (ED y FDS) para determinar el gesto realizado por la mano sana, con el fin de proporcionar la señal PWM adecuada a los actuadores del exoesqueleto para lograr el gesto objetivo de la mano parética. El módulo de reconocimiento de gestos procesa las señales EMG normalizadas utilizando el MVC cada $20 \mathrm{~ms}$ (período de actualización del valor de las señales EMG normalizadas) para determinar los tres gestos predefinidos. Esto depende de los valores de las señales EMG normalizadas (nEMG) y de los dos valores de los umbrales EMG, que se fijan en función de la calibración inicial de cada sujeto según su actividad muscular residual (Fig. 5). 


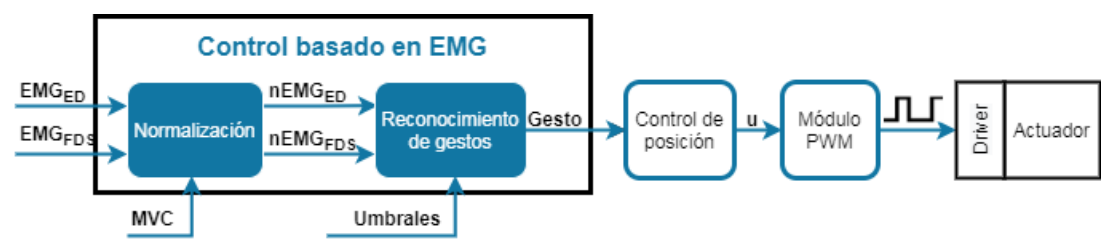

Figura 5: Diagrama del control por umbrales de EMG.

El gesto de mano cerrada se reconoce cuando la señal normalizada del músculo FDS supera el umbral de flexión $\mu$ mientras que la señal normalizada del músculo FDS es mayor que la señal normalizada del músculo ED y, en consecuencia, los actuadores se extienden, por lo que el exoesqueleto se cierra. Del mismo modo, el gesto de mano abierta se reconoce cuando la señal del músculo ED supera el umbral de extensión $\varepsilon$, mientras que la señal normalizada del músculo ED es mayor que la señal normalizada del músculo FDS, y los actuadores se retraen, por lo que el exoesqueleto se abre. El gesto de reposo se reconoce cuando ambas señales normalizadas de EMG son inferiores a sus respectivos umbrales y el exoesqueleto de mano alcanza el gesto de reposo (Fig. 6).

\section{ENTORNO SOFTWARE}

Se ha desarrollado un entorno software que proporciona soporte al terapeuta para la gestión de pacientes (Fig. 7.a) y terapias (Fig. 7.b). Mediante esta aplicación, el terapeuta selecciona el paciente que realiza la sesión y define la terapia a realizar por el mismo, de acuerdo con su historial clínico y de terapias realizadas.

En general, se ha demostrado que el uso de terapias de rehabilitación basados en entornos de realidad virtual de tipo videojuegos, también llamadas 'juegos serios', son menos aburridas que las terapias convencionales y son beneficiosas para la rehabilitación de pacientes que han sufrido un ictus $[5,17]$.

Con el objetivo de aumentar la efectividad del sistema de rehabilitación RobHand, se han desarrollado un conjunto de juegos serios para la realización de terapias bilaterales basadas en EMG. Los juegos han sido desarrollados en C\# utilizando los entornos de programación de Unity y Visual Studio.
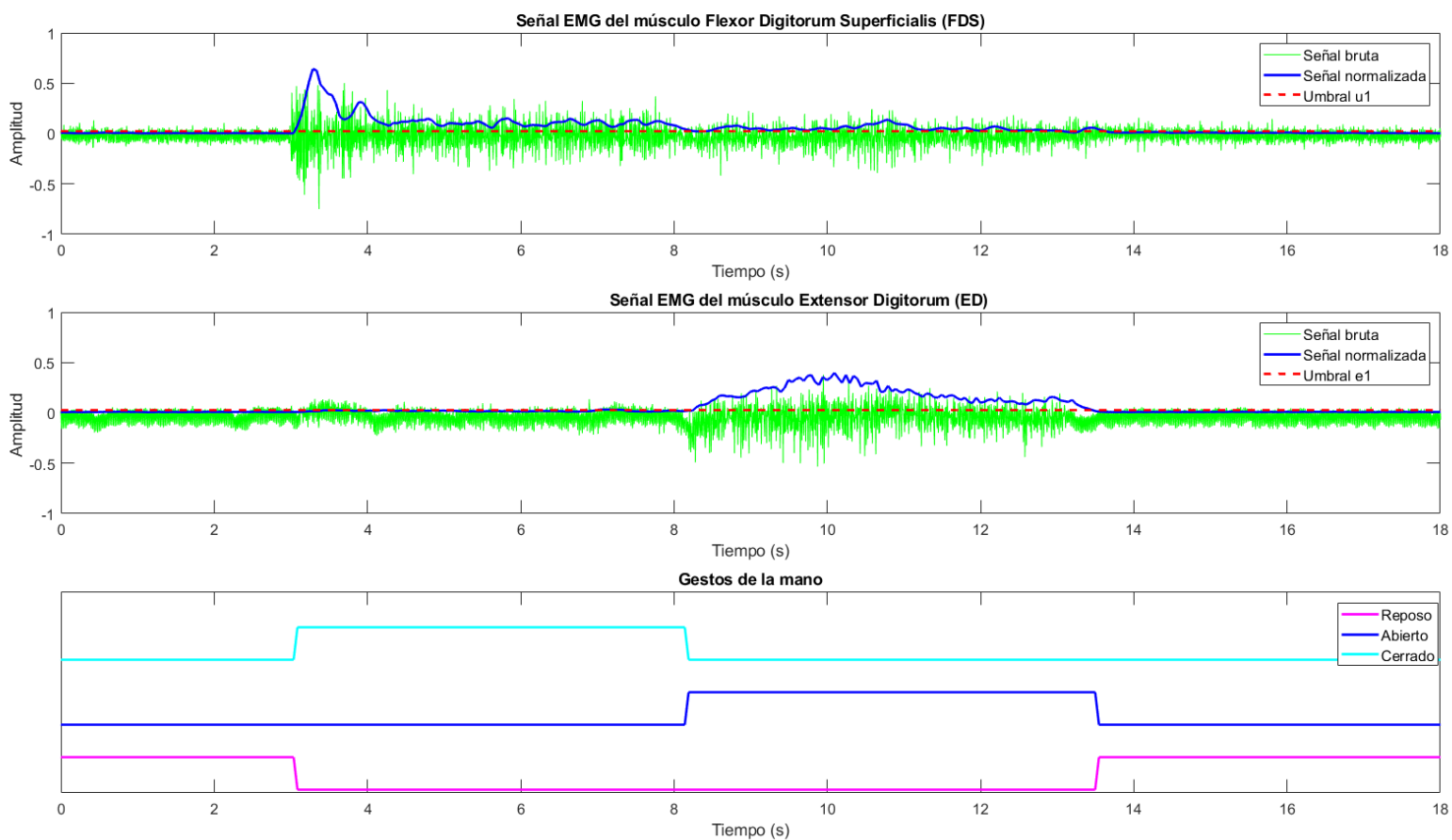

Figura 6: Tratamiento de las señales EMG en el control para el reconocimiento de gestos: señales en bruto de los músculos FDS y ED, señales normalizadas, umbrales y gestos identificados. 


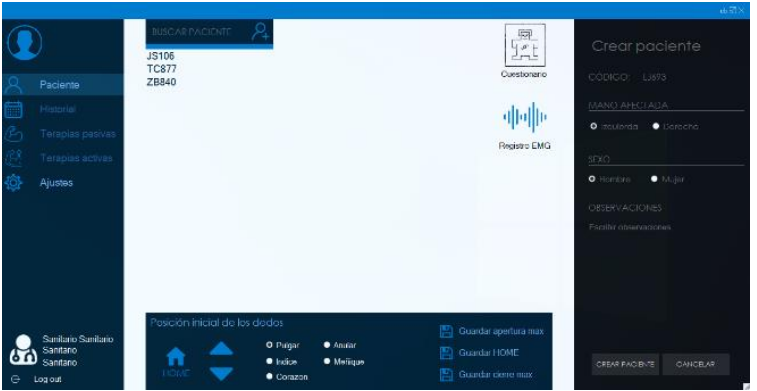

(a)

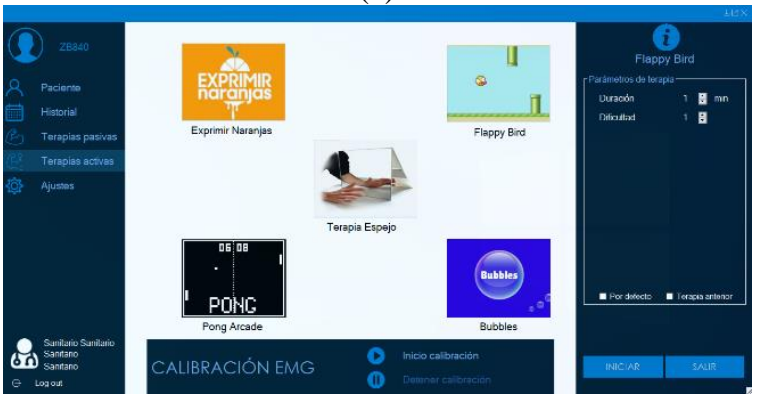

(b)

Figura 7: Entorno software. (a) Pantalla para la gestión de pacientes (b) Pantalla para la selección y parametrización de terapias bilaterales basadas en EMG utilizando entornos de realidad virtual.

Los juegos serios desarrollados son los siguientes: (i) Exprimir naranjas (ii) Flappy bird (iii) Pong (iv) Burbujas. La duración y la dificultad de las terapias son configurados por el personal sanitario. Todos los juegos proporcionan retroalimentación visual y sonora. También se ha incluido un feedback de recuperación comprensible para el usuario a través de la puntuación final del juego.

\section{CONCLUSIONES}

En este trabajo se ha desarrollado un sistema compacto de bajo coste que proporciona una solución embebida para el control en tiempo real de un exoesqueleto para rehabilitación de mano. La solución desarrollada cuenta con un sistema de adquisición de señales EMG de diseño propio y bajo coste que permite registrar señales de EMG. Además, el procesamiento de las señales EMG y el control se realizan en tiempo real en el microcontrolador, lo que reduce el tiempo de latencia del sistema, a la vez que proporciona un buen rendimiento para detectar la intención del usuario y replicar el movimiento identificado en el exoesqueleto de mano. Se ha implementado un control por umbrales para el reconocimiento de gestos porque su simplicidad permite implementarlo en un sistema embebido en tiempo real sin comprometer la fiabilidad.

Al diseñar y desarrollar el sistema de rehabilitación hemos intentado minimizar el tiempo de latencia en la medida de lo posible:
- El procesamiento digital de las señales EMG se realiza justo en el momento en que han sido muestreadas a $200 \mathrm{~Hz}$ por el conversor ADC y transmitidas al microcontrolador a través de comunicación SPI

- El alto rendimiento de la MCU TMS320F28069M, en concreto, la utilización el núcleo CLA para la implementación del procesamiento y el control de las señales EMG

- La optimización del algoritmo de rectificación de las señales EMG registradas con el sistema de adquisición de señales de diseño propio (selección de los filtros, del método de rectificación, de la ventana de tiempo...).

- Tanto el procesamiento de las señales de EMG como la generación de las señales de control de los actuadores es generada por el microcontrolador, por lo que no hay tiempo de latencia de transmisión a diferencia de trabajos anteriores, donde el procesamiento de EMG se realizaba en el PC por lo que era necesario transmitir los datos desde el PC al microcontrolador que controla los actuadores.

En resumen, se ha presentado una solución tecnológica de bajo coste que permite el control en tiempo real de un exoesqueleto mediante el análisis de las señales EMG. Permite la realización de terapias bilaterales de rehabilitación basadas en realidad virtual, ya que hacen posible que el exoesqueleto RobHand mueva la mano dañada reproduciendo el movimiento de la mano sana con un tiempo de respuesta y una precisión tolerables

\section{Agradecimientos}

El trabajo reflejado en esta comunicación está siendo realizado gracias a la financiación con fondos FEDER del Ministerio de Ciencia e Innovación, a través del proyecto Retos Investigación PID2019111023RB-C33, y del CDTI y la empresa CyL ImasD Informática S.L., mediante el proyecto IDI20170263.

\section{English summary}

INTEGRATED EMG-BASED CONTROL SOLUTION TO SUPPORT BILATERAL THERAPIES ON THE ROBHAND EXOSKELETON 


\begin{abstract}
Assisted bilateral rehabilitation has been proven to help patients improve their ability of the paretic limb and promote their motor recovery after suffering $a$ cerebrovascular accident (ACV), especially in upper limbs. Robotic-assisted bilateral rehabilitation based on EMG-driven control has been previously addressed in other studies to improve the hand mobility, but there is a lack of low-cost embedded solutions for the real-time bio-cooperative control of the robotic rehabilitation platforms. This paper presents a proprietary, low-cost, real-time EMG integrated solution implemented on the RobHand
\end{abstract}

[1] Ben I. A., Bouteraa Y., y Rekik C., (2017) "Design and development of $3 \mathrm{~d}$ printed myoelectric robotic exoskeleton for hand rehabilitation," Int. J. Smart Sens. Intell. Syst., vol. 10, no. 2, pp. 341-366.

[2] Burns M. K., Pei D., y Vinjamuri R., (2019) "Myoelectric control of a soft hand exoskeleton using kinematic synergies," IEEE Trans. Biomed. Circuits Syst., vol. 13, no. 6, pp. 13511361.

[3] Chen Y., Yang Z., y Wen Y., (2021) "A soft exoskeleton glove for hand bilateral training via surface EMG," Sensors, vol. 21, no. 2, pp. 118.

[4] Chowdhury R. H., Reaz M. B. I., Bin Mohd Ali M. A., Bakar A. A. A., Chellappan K., y Chang T. G., (2013) "Surface electromyography signal processing and classification techniques," Sensors, vol. 13, no. 9, pp. 12431-12466.

[5] Crosbie J. H. et al., (2008) "Virtual reality in the rehabilitation of the upper limb after hemiplegic stroke: a randomised pilot study," Screen, pp. 229-235.

[6] Fischer H. C., Stubblefield K., Kline T., Luo X., Kenyon R. V., y Kamper D. G., (2007) "Hand Rehabilitation Following Stroke: A Pilot Study of Assisted Finger Extension Training in a Virtual Environment," Top. Stroke Rehabil., vol. 14, no. 1, pp. 1-12.

[7] Halaki M. y Ginn K., (2012) "Normalization of EMG Signals: To Normalize or Not to Normalize and What to Normalize?," in Computational Intelligence in Electromyography Analysis - A perspective on Current Applications and Future Challenges, G. Naik, Ed, Croatia, InTech, pp. 175-194.

[8] Johnson, W., Onuma O., Owolabi M., y Sachdev S., (2016) "Stroke: a global response is needed," Bull. World Health Organ., vol. 94, no. 9, pp. 634-634.

[9] Leonardis D. et al., (2015) "An EMGcontrolled robotic hand exoskeleton for bilateral rehabilitation," IEEE Trans. Haptics, vol. 8, no. 2, pp. 140-151.
(Robot for Hand Rehabilitation) platform, an exoskeleton that supports bilateral EMG-based therapies. A threshold non-pattern recognition EMGdriven control for RobHand has been developed, which detects the hand gesture of the healthy hand and replicates it on the exoskeleton placed on the paretic hand.

Keywords: Electromyography, Control, Exoskeletons, Neurorehabilitation, Rehabilitation robotics.

\section{Referencias}

[10] Lu Z., Chen X., Zhang X., Tong K. Y., y Zhou P., (2017) "Real-Time Control of an Exoskeleton Hand Robot with Myoelectric Pattern Recognition," Int. J. Neural Syst., vol. 27, no. 5, pp. 1-11.

[11] Moreno-San Juan V., Cisnal A., Fraile J., PérezTuriel J., y de la Fuente E., (2021) "Design and Characterization of a Lightweight Underactuated RACA Hand Exoskeleton for Neurorehabilitation," in Robot Auton. Syst.

[12] Park S. et al., (2020) "User-Driven Functional Movement Training with a Wearable Hand Robot after Stroke," IEEE Trans. Neural Syst. Rehabil. Eng., vol. 28, no. 10, pp. 2265-2275.

[13] Park S., Meeker C., Weber L. M., Bishop L., Stein J., y Ciocarlie M., (2018) "Multimodal sensing and interaction for a robotic hand orthosis," IEEE Robot. Autom. Lett., vol. 4, no. 2, pp. 315-322.

[14] Rathore, S. S., Hinn, A. R., Cooper L. S., Tyroler H. A., y Rosamond W. D., (2002) "Characterization of incident stroke signs and symptoms: findings from the atherosclerosis risk in communities study.," Stroke, vol. 33, no. 11, pp. 2718-21.

[15] Secciani N., Topini A., Ridolfi A., Meli E., y Allotta B., (2020) "A novel Point-in-Polygonbased sEMG classifier for Hand Exoskeleton Systems," IEEE Trans. Neural Syst. Rehabil. Eng., vol. XX, no. XX, pp. 1-9.

[16] Sterr A. y Freivogel S., (2003) "Motorimprovement following intensive training in low-functioning chronic hemiparesis.," Neurology, vol. 61, no. 6, pp. 842-4.

[17] Subramanian S. K., Lourenço C. B., Chilingaryan G., Sveistrup H., y Levin M. F., (2013) "Arm motor recovery using a virtual reality intervention in chronic stroke: Randomized control trial," Neurorehabil. Neural Repair, vol. 27, no. 1, pp. 13-23.

[18] Ueki S. et al., "Development of a hand-assist robot with multi-degrees-of-freedom for rehabilitation therapy," IEEE/ASME Trans. Mechatronics, vol. 17, no. 1, pp. 136-146, 2012. 
[19] Wu H. et al., (2011) "Virtual reality based robotic therapy for stroke rehabilitation: An initial study," 2011 IEEE ICMA, Beijing, CN., pp. 1196-1200.

[20] Zhang J., Wang B., Zhang C., Xiao Y, y Wang M. Y., (2019) "An EEG/EMG/EOG-based multimodal human-machine interface to realtime control of a soft robot hand," Front. Neurorobot., vol. 13.

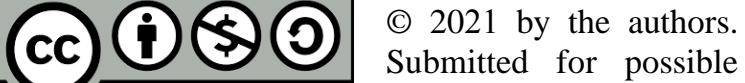
BY NC SA open access publication under the terms and conditions of the Creative Commons Attribution CC BY-NC-SA 4.0 license (https://creativecommons.org/licenses/by-ncsa/4.0/deed.es). 